\title{
Efecto diurético agudo de los extractos etanólico y acuoso de Ceratopteris pteridoides (Hook) en ratas normales
}

\author{
Antistio Aníbal Alviz, Rubén Darío Salas, Luis Alberto Franco \\ Grupo de Evaluación Biológica de Sustancias Promisorias, Facultad de Ciencias Farmacéuticas, \\ Universidad de Cartagena, Cartagena, Colombia
}

\begin{abstract}
Introducción. Ceratopteris pteridoides es un helecho semiacuático de la familia Parkeriacea, ampliamente utilizado en la medicina popular colombiana como diurético y colelitiásico, sobre el cual no existen reportes científicos que avalen su uso popular como diurético.

Objetivo. Evaluar el efecto diurético agudo en dosis única y dosis repetidas a corto plazo, de los extractos etanólico y acuoso de C. pteridoides en un modelo in vivo.

Materiales y métodos. El extracto etanólico total fue obtenido por maceración de la planta entera de $C$. pteridoides con etanol y el extracto acuoso fue obtenido por decocción a $60{ }^{\circ} \mathrm{C}$ por 15 minutos. Ambos extractos se sometieron a análisis fitoquímico preliminar y estudio histológico posterior a la administración de los extractos durante ocho días consecutivos (1.000 mg/kg). El efecto diurético se evaluó en ratas Wistar, tratadas con los extractos $(500 \mathrm{mg} / \mathrm{kg})$, en forma aguda y en dosis repetidas a corto plazo, cuantificando la eliminación de agua y la excreción renal de sodio y potasio por espectrofotometría de absorción atómica y, de cloruros, por titulación mercurimétrica.

Resultados. En el modelo agudo, ambos extractos mostraron un significativo efecto diurético y de excreción renal de sodio y potasio en comparación con el control, mientras que con la administración en dosis repetidas a corto plazo mostraron efecto diurético sin eliminación de electrolitos. El estudio histopatológico no sugirió efectos tóxicos hepáticos o renales.

Conclusión. Los resultados demuestran la actividad diurética de C. pteridoides y sustentan el uso popular dado a esta planta como diurético en la costa norte colombiana. Se requieren estudios posteriores que permitan aislar e identificar los compuestos responsables de la actividad y los mecanismos de acción involucrados.
\end{abstract}

Palabras clave: plantas medicinales, diuréticos, electrolitos, furosemida.

doi: http://dx.doi.org/10.7705/biomedica.v32i4.750

Acute diuretic effect of ethanolic and aqueous extracts of Ceratopteris pteridoides (Hook) in normal rats

Introduction. Ceratopteris pteridoides is a semiaquatic fern of the Parkeriacea family, widely used in the Colombian folk medicine as a diuretic and cholelithiasic, of which there are no scientific reports that validate its popular use.

Objective. To evaluate the acute and short-term repeated-dose diuretic effect of the ethanolic and aqueous extracts of $C$. pteridoides in an in vivo model.

Materials and methods. The total ethanolic extract was obtained by maceration of the whole plant of C. pteridoides with ethanol and the aqueous extract by decoction at $60^{\circ} \mathrm{C}$ for 15 minutes. Both extracts were evaluated in preliminary phytochemical analysis and histological studies after the administration of the extracts for 8 consecutive days $(1000 \mathrm{mg} / \mathrm{Kg})$. The diuretic effect was evaluated using Wistar rats treated with the extracts $(500 \mathrm{mg} / \mathrm{Kg})$, using an acute and a short-term repeated-dose model, and quantifying water elimination, sodium and potassium excretion by atomic absorption spectrophotometry, and chloride excretion by mercurimetric titration.

Results. In the acute model both extracts showed significant diuretic, natriuretic, and kaliuretic effect compared to the control group. Whereas, a short-term repeated-dose administration showed a diuretic effect without elimination of electrolytes. The histopathologic study did not suggest a toxic effect in liver or kidney.

Conclusion. The results represent evidence of the diuretic activity of $C$. pteridoides and give support the popular use given to this plant in the north coast of Colombia. Further studies are

Contribución de los autores:

Antistio Aníbal Alviz: participó en la ejecución de los ensayos de la actividad diurética.

Rubén Darío Salas: prestó la asesoría general en la ejecución de ensayos farmacológicos.

Luis Alberto Franco: prestó la asesoría general del trabajo de investigación y ejecución de ensayos farmacológicos. 
required to isolate and identify the compounds responsible for the activity and the mechanism of action involved.

Keywords: Plants, medicinal; diuretics, electrolytes, furosemide.

doi: http://dx.doi.org/10.7705/biomedica.v32i4.750

Los diuréticos se utilizan en medicina para los estados edematosos asociados a enfermedades como la hipertensión arterial sistémica, la falla cardiaca, la nefritis, la ascitis, el edema pulmonar y el edema cerebral (1-4). Sin embargo, algunos diuréticos ampliamente utilizados, como la furosemida y la hidroclorotiacida, se han asociado con efectos adversos como desequilibrio hidroelectrolítico, alteraciones metabólicas, desarrollo de nuevos casos de diabetes, activación del sistema neuroendocrino renina-angiotensina-aldosterona, deterioro de la función sexual y disfunción renal (5-7). En este sentido, aún existe la necesidad de buscar nuevos agentes diuréticos con menores efectos secundarios, que incrementen el arsenal terapéutico y que sean, además, de fácil acceso para las poblaciones marginadas de bajos recursos.

El uso actual dado a las plantas medicinales para el tratamiento de diversas enfermedades conocidas, ha renovado en la mayoría de los países en desarrollo, así como en los países industrializados, un enorme interés por los productos naturales, ya que constituyen una importante fuente de moléculas potencialmente activas, teniendo en cuenta la gran diversidad química y el hecho de que, aproximadamente, la mitad de los fármacos utilizados actualmente en medicina tienen un origen natural, proporción que alcanza el $75 \%$ en el caso de los que se emplean para los trastornos cardiovasculares y renales $(4,8,9)$.

Ceratopteris pteridoides es un helecho flotante o semiacuático de la familia Parkeriaceae (10), utilizado en la medicina popular de la costa norte colombiana como diurético y colelitiásico. En el campo fitoquímico, este género ha sido poco estudiado, encontrándose un solo reporte para la especie C. thalictroides, en el cual se evidenció la presencia de ácidos grasos insaturados del tipo hexadecanoico, mirístico, oleico, linoleico, palmítico y esteárico (11). Desde el punto de vista

Correspondencia:

Luis Alberto Franco, Facultad de Ciencias Farmacéuticas, Universidad de Cartagena sede Zaragocilla, Cartagena de Indias, Colombia.

Teléfono: (575) 669 8323; fax (575) 6698278

Ifrancoo@unicartagena.edu.co

Recibido: 01/12/11; aceptado:11/10/12 farmacológico, no se dispone de reportes científicos que avalen el uso popular dado a esta planta.

Este estudio se realizó con el objeto de evaluar el efecto diurético agudo, con dosis única y con dosis repetidas a corto plazo, de los extractos etanólico y acuoso de C. pteridoides en un modelo in vivo, empleando ratas como animales de investigación, y se constituye en la primera investigación sobre la eficacia de esta planta como agente diurético.

\section{Materiales y métodos \\ Obtención de los extractos}

El material vegetal de C. pteridoides se recolectó en el municipio de San Bernardo del Viento (Córdoba). La planta fue clasificada botánicamente por el Herbario de la Universidad de Antioquia y una muestra quedó depositada en sus archivos como referencia (HUA166134).

El material vegetal seco y pulverizado de C. pteridoides se sometió a maceración hasta agotamiento con etanol al $96 \%$. El filtrado se concentró en rotaevaporador con calentamiento suave y presión reducida y se identificó como extracto etanólico total (Cp-EtOH). Otra porción del material vegetal seco y pulverizado de esta planta se sometió a decocción por 15 minutos a $60^{\circ} \mathrm{C}$; el filtrado de esta decocción posteriormente fue congelado, liofilizado y catalogado como extracto total acuoso $\left(\mathrm{Cp}-\mathrm{H}_{2} \mathrm{O}\right)$.

\section{Estudio fitoquímico}

Con el fin de establecer los grupos de metabolitos secundarios mayoritarios, los extractos totales de $C$. pteridoides se sometieron a un análisis fitoquímico preliminar basado en reacciones de precipitación o de coloración, que contó con pruebas para flavonoides, leucoantocianinas, saponinas, taninos, terpenos, quinonas, alcaloides, aminas, triptaminas y compuestos fenólicos.

\section{Determinación del efecto diurético}

Se utilizaron ratas Wistar hembra suministradas por el Instituto Nacional de Salud, con peso entre 150 y $200 \mathrm{~g}$, las cuales se mantuvieron en condiciones ambientales estándar $\left(25 \pm 1{ }^{\circ} \mathrm{C}, 55 \pm 5 \%\right.$ de humedad y ciclos de luz-oscuridad de 12 horas), con alimentación y agua ad libitum. Los experimentos fueron realizados dando cumplimiento a lo dispuesto en la Ley 84 de 1989, la Resolución Nº 008430 de 
1993 y las recomendaciones internacionales para el manejo de animales de experimentación.

Para la evaluación del efecto diurético, se siguió el método descrito por Kau, et al., en 1984, con pequeñas modificaciones (12). Las ratas fueron colocadas en cajas metabólicas individuales, 24 horas antes de iniciar el experimento, para su adaptación. Tanto en el ensayo agudo con dosis única como en aquel con dosis repetidas a corto plazo, los animales se separaron al azar en cuatro grupos de cinco ratas cada uno $(n=5)$. Los animales se privaron de alimentación durante ocho horas antes de cada ensayo, y de agua, al inicio del mismo.

En el ensayo agudo, antes de la administración de los tratamientos, todos los animales recibieron una dosis oral de solución salina al 0,9\% (2 ml/100 $\mathrm{g}$ de peso corporal), para establecer una carga uniforme de agua y sal (13). Inmediatamente después, los grupos de animales fueron tratados por vía oral con dosis única de los extractos de $C$. pteridoides $(500 \mathrm{mg} / \mathrm{kg})$, furosemida $(10 \mathrm{mg} / \mathrm{kg}$ ) como control positivo y vehículo (agua $2 \mathrm{ml} / 100 \mathrm{~g}$ de peso) como control negativo, respectivamente. La orina se recolectó durante ocho horas después de la administración de la dosis.

El ensayo con dosis repetidas a corto plazo implicó la administración de una dosis diaria de los extractos de C. pteridoides $(500 \mathrm{mg} / \mathrm{kg}$ ) y furosemida $(10 \mathrm{mg} /$ $\mathrm{kg}$ ) durante un periodo de ocho días consecutivos. El grupo control recibió agua (2 $\mathrm{ml} / 100 \mathrm{~g}$ de peso) cada día. Al octavo día, una vez administrada la última dosis, los animales se colocaron en cajas metabólicas individuales y se recolectó la orina de ocho horas. Las ratas se observaron diariamente, con el fin de evidenciar toxicidad aparente.

En ambos ensayos, se midió el volumen total de orina excretada en el intervalo de tiempo (8 horas) y se determinó en ella el pH, y la concentración de sodio $\left(\mathrm{Na}^{+}\right)$, de potasio $\left(\mathrm{K}^{+}\right)$y de cloruro $\left(\mathrm{Cl}^{-}\right)$. $\mathrm{La}$ excreción de orina acumulada se calculó en relación con el peso corporal y se expresó en ml/100 g. Los electrolitos sodio y potasio se determinaron por espectrofotometría de absorción atómica, utilizando un equipo de absorción atómica Solar-Unicam $969^{\mathrm{TM}}$, el ión cloruro, por valoración mercurimétrica en presencia de indicador mixto de bromofenol azuldifenilcarbazona, y se expresaron en relación con el peso corporal como $\mathrm{mEq} / 100 \mathrm{~g}$ (14).

\section{Estudio histológico}

Para evaluar el efecto hepático y renal de los extractos de C. pteridoides, se hizo un análisis histológico, después de la administración oral durante ocho días consecutivos, de una dosis de $1.000 \mathrm{mg} / \mathrm{kg}$ de peso. Se fijaron en formol al $10 \%$ en PBS por 24 horas, cortes de hígado y de riñón de los animales; se deshidrataron en etanol y se embebieron en parafina. Se cortaron secciones de $5 \mu \mathrm{m}$ de espesor de los tejidos, utilizando un micrótomo (Leica RM $2125^{\mathrm{TM}}$ ), las cuales se colocaron en láminas de vidrio y se secaron toda la noche a $37^{\circ} \mathrm{C}$. Posteriormente, estas secciones se limpiaron, se hidrataron y se fijaron con hematoxilina y eosina, de acuerdo con los protocolos estandarizados (15). Las láminas se codificaron para evitar sesgos durante la evaluación y se examinaron en un microscopio Nikon Eclipse $\mathrm{E} 400^{\mathrm{TM}}$, para caracterizar los cambios histopatológicos.

\section{Análisis estadístico}

Los valores en las figuras y textos son expresados como la media aritmética \pm error estándar de la media (ESM). Las diferencias entre los grupos se determinaron mediante análisis de varianza de una vía (ANOVA), seguido de pruebas de Dunnet y Tukey como para hacer comparaciones múltiples. Los valores de $p$ menores de 0,05 se consideraron estadísticamente significativos.

\section{Resultados \\ Estudio preliminar fitoquímico}

Los resultados del análisis preliminar fitoquímico de los extractos de C. pteridoides evidenciaron abundantes aminas aromáticas y triptaminas, ésteres, aldehídos y cetonas, y en menor medida, se observaron taninos y cardiotónicos (cuadro 1).

\section{Efecto diurético de los extractos y la furosemida en dosis única}

El tratamiento con una sola dosis de $500 \mathrm{mg} /$ $\mathrm{kg}$ de los extractos etanólico y acuoso de $C$.

Cuadro 1. Estudio preliminar fitoquímico de los extractos de Ceratopteris pteridoides

\begin{tabular}{lcc}
\hline \multirow{2}{*}{ Metabolitos } & \multicolumn{2}{c}{ Extracto } \\
secundarios & Etanólico & Acuoso \\
\hline Alcaloides & - & - \\
Cardiotónicos & + & + \\
Aldehídos y cetonas & +++ & +++ \\
Aminas y triptaminas & +++ & +++ \\
Ésteres & +++ & +++ \\
Quinonas & - & - \\
Flavonoides & - & - \\
Taninos & ++ & ++ \\
Saponinas & - & - \\
Cumarinas & - & - \\
\hline
\end{tabular}

(+++): abundante; (++): moderado; (+): leve; (-): no detectado. 
pteridoides, incrementó el volumen excretado de orina, con valores similares en ambos extractos y significativamente superiores a los presentados en el grupo control $(p<0,001)$, lo que constituye un importante efecto inductor de la excreción de agua (cuadro 2). En cuanto a la excreción de electrolitos, los extractos de $C$. pteridoides aumentaron significativamente la eliminación renal de sodio, potasio y cloruro, en comparación con el grupo control; el extracto etanólico fue más activo y aumentó la eliminación de los electrolitos $\mathrm{Na}^{+}$y Cl- $(p<0,001)$, con valores similares a los producidos por la furosemida utilizada como control. También, se observó que tanto la infusión como el extracto etanólico de esta planta, incrementaron la excreción de potasio, pero en proporción significativamente menor a la producida por la furosemida (figura 1).

\section{Efecto diurético de los extractos y la furosemida en dosis repetidas}

La administración de dosis durante ocho días consecutivos de los extractos etanólico y acuoso de
C. pteridoides $(500 \mathrm{mg} / \mathrm{kg}$ ), produjo un significativo incremento en el volumen excretado de orina $(p<0,001)$, comparable al efecto producido por la furosemida, y presentaron un comportamiento similar al presentado en el ensayo agudo (cuadro 3). En cuanto a la excreción de electrolitos, con el extracto etanólico se observó una considerable disminución en la excreción de sodio, potasio y cloruro, en comparación con el efecto producido en el ensayo agudo y con valores significativamente menores a los presentados con la furosemida.

Por su parte, el extracto acuoso bajo este esquema de administración, no indujo la eliminación renal de $\mathrm{Na}^{+}, \mathrm{K}^{+}$y $2 \mathrm{Cl}^{-}$(figura 1). El efecto ahorrador de electrolitos observado con ambos extractos de C. pteridoides es particularmente importante en la excreción de $\mathrm{K}^{+}$, lo que sugiere una posible acción como diuréticos ahorradores de potasio. Estos datos demuestran que la administración de dosis repetidas a corto plazo de los extractos de esta planta, produce un importante efecto acuarético, con pobre eliminación de electrolitos.

Cuadro 2. Efecto diurético agudo de los extractos de Ceratopteris pteridoides en ratas normales

\begin{tabular}{|c|c|c|c|c|}
\hline Tratamiento & $\begin{array}{c}\text { Orina excretada } \\
\mathrm{ml} / 100 \mathrm{~g}\end{array}$ & $\begin{array}{c}{\left[\mathrm{Na}^{+}\right]} \\
\mathrm{mEq} / 100 \mathrm{~g}\end{array}$ & $\begin{array}{c}{\left[\mathrm{K}^{+}\right]} \\
\mathrm{mEq} / 100 \mathrm{~g}\end{array}$ & $\begin{array}{c}{[\mathrm{Cl}-]} \\
\mathrm{mEq} / 100 \mathrm{~g}\end{array}$ \\
\hline Control & $2,84 \pm 0,125$ & $0,17 \pm 0,006$ & $0,07 \pm 0,006$ & $0,50 \pm 0,005$ \\
\hline Furosemida & $5,50 \pm 0,078^{\star * *}$ & $0,34 \pm 0,020^{* * *}$ & $0,23 \pm 0,008^{\star * *}$ & $0,97 \pm 0,014^{* * *}$ \\
\hline Extracto etanólico & $4,18 \pm 0,061^{\star * *}$ & $0,34 \pm 0,012^{* * *}$ & $0,14 \pm 0,010^{\star * *}$ & $0,92 \pm 0,035^{\star \star *}$ \\
\hline Extracto acuoso & $3,90 \pm 0,080^{\star * *}$ & $0,35 \pm 0,008^{\star \star *}$ & $0,12 \pm 0,010^{* *}$ & $0,78 \pm 0,008^{* * *}$ \\
\hline
\end{tabular}

Los valores representan la media $\pm E S M, n=5$ en cada grupo

Análisis estadístico mediante ANOVA de una vía seguido de un test de Dunnett para comparaciones múltiples.

${ }^{* *} p<0.01 ;{ }^{* * *} p<0.001$, estadísticamente significativo frente al grupo control.

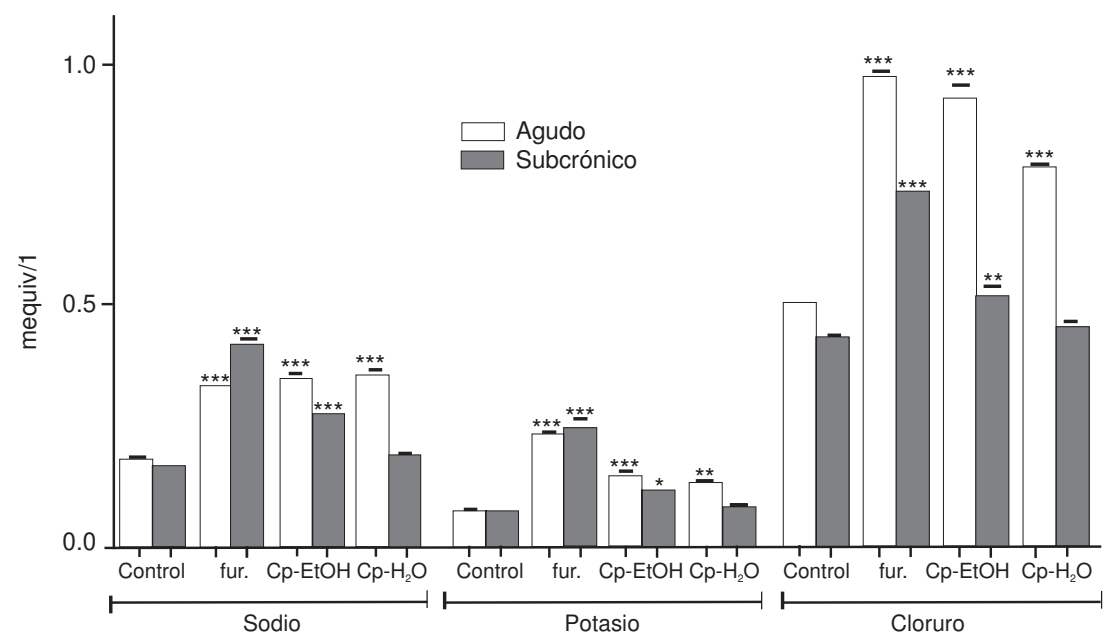

Figura 1. Efecto agudo y de dosis repetidas a corto plazo de los extractos de $C$. pteridoides y furosemida sobre la excreción de los electrolitos $\mathrm{Na}^{+}, \mathrm{K}^{+}, \mathrm{Cl}$. Los valores representan la media $\pm \mathrm{ESM}$, de cinco animales por grupo.

${ }^{*} p<0,05 ;{ }^{* *} p<0,01 ;{ }^{* * *} p<0,001$, estadísticamente significativo frente al grupo control 


\section{Estudio histológico}

El estudio histopatológico mostró que la administración repetida de los extractos etanólico y acuoso de C. pteridoides (1.000 mg/kg de peso por vía oral), no produjo alteraciones significativas hepáticas ni renales. Específicamente en el riñón, no se observó degeneración del tejido epitelial tubular, y no hubo infiltración de células mononucleares en la médula ni en la corteza de los epitelios tubulares con ambos extractos; solo con el extracto acuoso se observó una leve congestión medular en los vasos renales, pero sin compromiso tubular. Estos resultados

Cuadro 3. Efecto diurético de dosis repetidas a corto plazo de los extractos de Ceratopteris pteridoides en ratas normales

\begin{tabular}{|c|c|c|c|c|}
\hline Tratamiento & $\begin{array}{c}\text { Orina excretada } \\
\mathrm{ml} / 100 \mathrm{~g}\end{array}$ & $\begin{array}{c}{\left[\mathrm{Na}^{+}\right]} \\
\mathrm{mEq} / 100 \mathrm{~g}\end{array}$ & $\begin{array}{c}{\left[\mathrm{K}^{+}\right]} \\
\mathrm{mEq} / 100 \mathrm{~g}\end{array}$ & $\begin{array}{c}{[\mathrm{Cl}]} \\
\mathrm{mEq} / 100 \mathrm{~g}\end{array}$ \\
\hline Control & $3,10 \pm 0,052$ & $0,16 \pm 0,002$ & $0,07 \pm 0,002$ & $0,43 \pm 0,011$ \\
\hline Furosemida & $6,00 \pm 0,076^{* * *}$ & $0,41 \pm 0,020^{* * *}$ & $0,24 \pm 0,020^{\star * *}$ & $0,73 \pm 0,014^{* * *}$ \\
\hline Extracto etanólico & $5,46 \pm 0,049^{\star * *}$ & $0,27 \pm 0,004^{\star * *}$ & $0,11 \pm 0,005^{\star}$ & $0,52 \pm 0,022^{* *}$ \\
\hline Extracto acuoso & $4,66 \pm 0,060^{\star * *}$ & $0,19 \pm 0,003$ & $0,08 \pm 0,004$ & $0,45 \pm 0,013$ \\
\hline
\end{tabular}

Los valores representan la media $\pm E S M, n=5$ en cada grupo.

Análisis estadístico mediante ANOVA de una vía seguido de un test de Dunnett para comparaciones múltiples.

${ }^{*} \mathrm{p}<0,05 ;{ }^{* *} \mathrm{p}<0,01 ;{ }^{* *} \mathrm{p}<0,001$, estadísticamente significativo frente al grupo control.
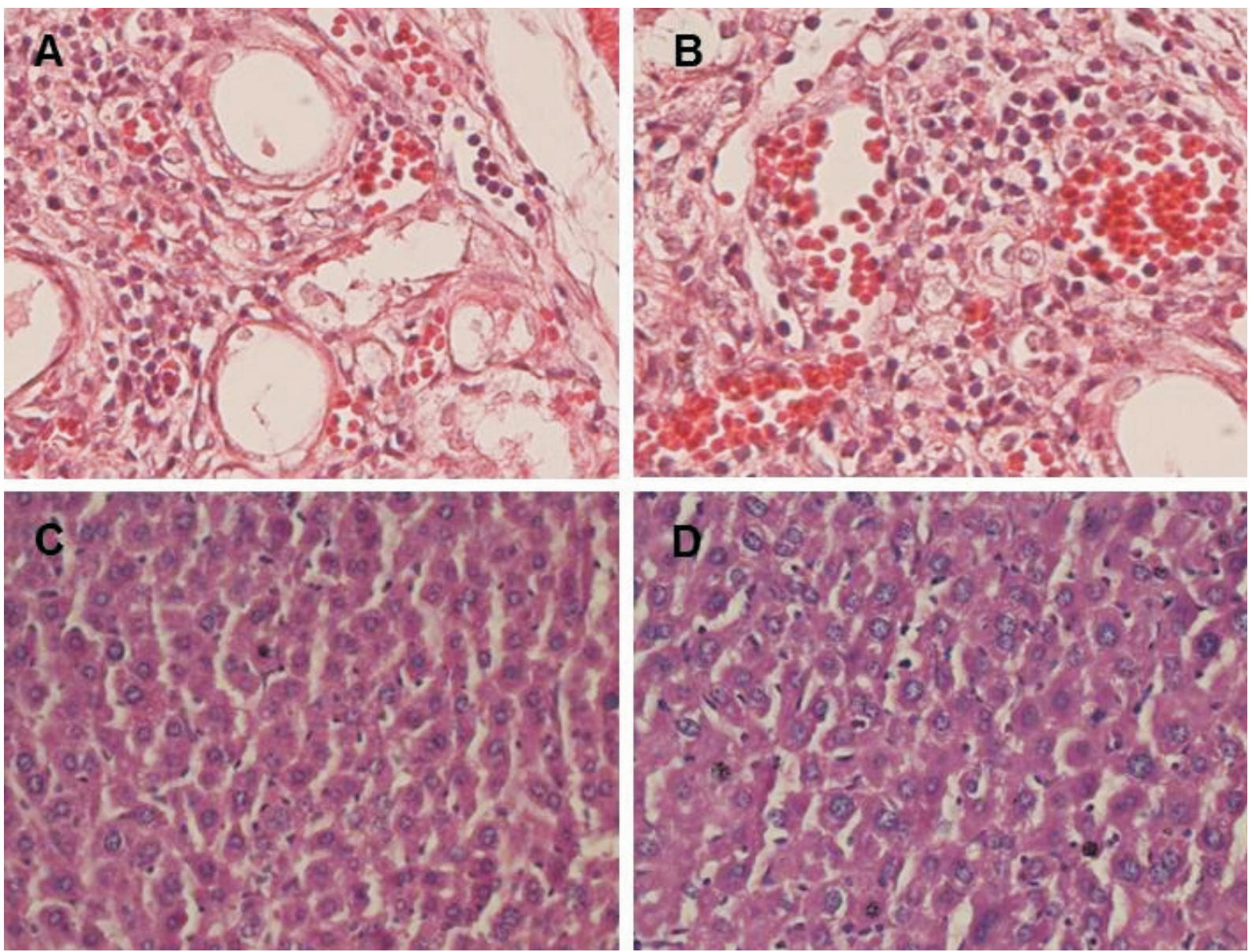

Figura 2. En los grupos tratados con extracto etanólico (A) y extracto acuoso (B) de C. pteridoides; no se observa en el riñón, degeneración del tejido epitelial tubular, no hay infiltración de células mononucleares en la médula ni en la corteza de los epitelios tubulares; en el grupo con extracto acuoso se observa congestión medular en los vasos renales, pero sin compromiso tubular. En el tejido hepático de los grupos con extracto etanólico (C) y extracto acuoso (D), se observan la vena porta y la arteria hepática normales, sin aumento de células de Kupffer, no hay inflamación ni vacuolización, y los hepatocitos se observan sanos. Hematoxilina y eosina, 40X. 
permiten descartar posibles efectos tóxicos inducidos por los extractos acuoso y etanólico de esta planta (figura 2).

\section{Discusión}

En el presente estudio, se evaluó el efecto diurético de los extractos etanólico y acuoso de $C$. pteridoides en ratas normales, después de la administración de dosis única de $500 \mathrm{mg} / \mathrm{kg}$ de peso por vía oral y después de la administración de la misma dosis durante ocho días consecutivos. Se eligió la vía de administración oral, dado que es la utilizada en la medicina tradicional. El efecto farmacológico se comparó con el producido por la furosemida, diurético de asa ampliamente utilizado en la práctica clínica y en los estudios de evaluación de la actividad diurética, el cual inhibe el cotransportador $\mathrm{Na}^{+}-\mathrm{K}^{+}-2 \mathrm{Cl}^{-}$situado en la porción gruesa del asa de Henle, inhibiendo la reabsorción de los electrolitos (4,7,16-18).

El tratamiento previo de los animales con una carga de solución salina representa una ventaja y es necesario para producir un aceptable grado de diuresis. Además, como los diuréticos son utilizados para el tratamiento del edema, es de gran importancia demostrar su efectividad en presencia de electrolitos y agua. De esta manera y acorde con lo establecido por Nedi, et al. (19), el exceso de agua y electrolitos suministrado a los animales, simula estas condiciones edematosas.

Los resultados obtenidos con los extractos etanólico y acuoso de $C$. pteridoides constituyen una base científica que apoya el uso diurético tradicionalmente dado a esta especie en poblaciones de la costa norte colombiana.

En el ensayo agudo, los extractos produjeron un significativo incremento en la excreción de la carga salina administrada y en la excreción urinaria de los electrolitos $\mathrm{Na}^{+}-\mathrm{K}^{+}-\mathrm{Cl}^{-}$, con un comportamiento similar al producido por la furosemida, con excepción de la excreción de potasio, la cual fue significativamente menor. Por su parte, la administración continua de estos extractos durante ocho días consecutivos, conduce a una significativa disminución en la excreción de electrolitos, especialmente del potasio, lo que indica que estos extractos probablemente no se comportan como diuréticos de asa y sugiere un interesante efecto ahorrador de potasio similar al mostrado por diuréticos de tipo amiloride y espirinolactona.
Por otro lado, con el extracto acuoso se observó una disminución en la eliminación de sodio, con un buen perfil de eliminación de agua, lo que sugiere un comportamiento similar al de los diuréticos de tipo antagonistas de los receptores de vasopresina V2, reportados por Hirano, et al., y posteriormente, por Schrier, et al., $(20,21)$. Además, el efecto de este extracto acuoso podría compararse con el de los diuréticos análogos del péptido natriurético auricular de tipo $\beta$ (PNA- $\beta$ ), reportados por Cobo, et al. (22). Estas diferencias en la excreción de electrolitos en los ensayos agudo con dosis única y con dosis repetidas a corto plazo, pueden explicarse por la compleja composición de los extractos evaluados, constituidos por numerosos metabolitos que pueden actuar a distintos niveles.

Entre los principales grupos de metabolitos identificados en los extractos de $C$. pteridoides, se encuentran aminas y triptaminas, ésteres, aldehídos y cetonas, y en menor proporción, algunos taninos y cardiotónicos, lo cual guarda relación con lo reportado en la literatura científica respecto a la composición de los helechos de este género $(11,23)$. El efecto diurético observado con los extractos de esta planta está relacionado con la presencia de estos constituyentes y se requieren posteriores estudios que permitan determinar cuáles son los responsables de la actividad y, asimismo, profundizar en sus mecanismos de acción.

La administración de los extractos etanólico y acuoso de C. pteridoides $(1.000 \mathrm{mg} / \mathrm{kg}$ por vía oral) durante ocho días consecutivos no produjo signos de toxicidad relevante en los animales de experimentación; tampoco ocasionó daños histopatológicos significativos en el hígado o el riñón, lo que nos permite descartar la posibilidad de efectos tóxicos inducidos por la administración de los extractos de esta planta.

En conclusión, el presente estudio proporciona una base cuantitativa que apoya el uso popular como diurético de $C$. pteridoides en la medicina tradicional. La infusión acuosa proporciona un efecto diurético similar al presentado por el extracto obtenido por maceración con etanol, lo cual sugiere que el uso de la infusión en la medicina popular, tal como se realiza actualmente, es adecuado y no es necesario reemplazarlo por un método de extracción química más costoso. Se requieren posteriores estudios experimentales encaminados a la identificación de los componentes responsables de la actividad 
y al entendimiento de los mecanismos de acción involucrados.

\section{Agradecimientos}

Los autores agradecen a la Universidad de Cartagena por la financiación de este trabajo. Extendemos nuestra gratitud a la Facultad de Ciencias Farmacéuticas por su apoyo general, a la Facultad de Medicina por su cooperación con el estudio histológico y a la química farmacéutica Yanet Ocampo Buendía por su colaboración en la revisión final del documento.

\section{Conflictos de intereses}

Los autores declaran no tener ningún conflicto de interés.

\section{Financiación}

El trabajo fue llevado a cabo con el apoyo financiero de la Vicerrectoría de Investigaciones de la Universidad de Cartagena (Proyecto código 068 de 2009).

\section{Referencias}

1. Chobanian AV, Bakris GL, Black HR, Cushman WC, Green LA, Izzo JL, et al. Seventh report of the joint national committee on prevention, detection, evaluation, and treatment of high blood pressure. Hypertension. 2003;42:1206-52. http://dx.doi.org/10.1161/01.HYP.0000107251.49515.c2

2. Jentzer JC, DeWald TA, Hernández AF. Combination of loop diuretics with thiazide-type diuretics in heart failure. J Am Coll Cardiol. 2010;56:1527-34. http://dx.doi. org/10.1016/j.jacc.2010.06.034,

3. Lacourcière $\mathbf{Y}$, Poirier L, Lefebvre J, Ross SA, Leenen FH. Increasing the doses of both diuretics and angiotensin receptor blockers is beneficial in subjects with uncontrolled systolic hypertension. Can J Cardiol. 2010;26:e313-9.

4. Sadki C, Hacht B, Souliman A, Atmani F. Acute diuretic activity of aqueous Erica multiflora flowers and Cynodon dactylon rhizomes extracts in rats. J Ethnopharmacol. 2010;128:352-6. http://dx.doi.org/10.1016/j.jep.2010.01.048

5. Gupta S, Neyses L. Diuretic usage in heart failure: A continuing conundrum in 2005. Eur Heart J. 2005;26:644-9. http://dx.doi.org/10.1093/eurheartj/ehi176

6. Morganti A. Should a diuretic always be the first choice in patients with essential hypertension? The case for no. J Am Soc Nephrol. 2005;16(Suppl.1):S70-3. http://dx.doi. org/10.1681/ASN.2004110964

7. Cleland JGF, Coletta A, Witte K. Practical applications of intravenous diuretic therapy in decompensated heart failure. Am J Med. 2006;119:S26-36. http://dx.doi.org/10.1016/j. amjmed.2006.09.014
8. Clark AM. Natural products as a resource for new drugs. Pharm Res. 1996;13:1133-41.

9. Newman DJ, Cragg GM, Snader KM. Natural products as sources of new drugs over the period 1981-2002. J Nat Prod. 2003;66:1022-37. http://dx.doi.org/10.1021/np030096|

10. Delascio Chitty F. Helechos acuáticos del Estado de Cojedes (Venezuela). An Jard Bot Madr. 1980;36:61-7.

11. Gemmrich AR. Fatty acid composition of fern spore lipids. Phytochemistry. 1977;16:1044-6.

12. Kau S, Keddie J, Andrews D. A method for screening diuretic agents in the rat. J Pharmacol Methods. 1984;11:6775. http://dx.doi.org/10.1016/0160-5402(84)90054-8

13. Lahlou S, Tahraoui A, Israili Z, Lyoussi B. Diuretic activity of the aqueous extracts of Carum carvi and Tanacetum vulgare in normal rats. J Ethnopharmacol. 2007;110:458-63. http://dx.doi.org/10.1016/j.jep.2006.10.005

14. Martín-Herrera D, Abdala S, Benjumea D, Pérez-Paz P. Diuretic activity of Withania aristata: An endemic Canary Island species. J Ethnopharmacol. 2007;113:487-91. http:// dx.doi.org/10.1016/j.jep.2007.07.005

15. Allen T, Prophet E. Métodos histotecnológicos: hematoxilina y eosina. Washington, D.C.: Instituto de Patología de las Fuerzas Armadas (AFIP); 1995. p. 55-60.

16. Shah SU, Anjum S, Littler WA. Use of diuretics in cardiovascular diseases: (1) Heart failure. Postgrad Med J. 2004;80:201-5. http://dx.doi.org/10.1136/pgmj.2003.010835

17. Kalant H, Roschlau WHE, Monsiváis RIS, Ackermann U. Principios de farmacología médica. 6a edición. Oxford: Universidad Iberoamericana; 2002. p. 525-39.

18. Rosner MH, Gupta R, Ellison D, Okusa MD. Management of cirrhotic ascites: Physiological basis of diuretic action. Eur J Intern Med. 2006;17:8-19. http://dx.doi.org/10.1016/j. ejim.2005.08.003

19. Nedi T, Mekonnen N, Urga K. Diuretic effect of the crude extracts of Carissa edulis in rats. J Ethnopharmacol. 2004;95:57-61. http://dx.doi.org/10.1016/j.jep.2004.06.017,

20. Hirano T, Yamamura Y, Nakamura S, Onogawa T, Mori T. Effects of the V2-receptor antagonist OPC-41061 and the loop diuretic furosemide alone and in combination in rats. $J$ Pharmacol Exp Ther. 2000;292:288-94.

21. Schrier RW, Gross P, Gheorghiade M, Berl T, Verbalis JG, Czerwiec FS, et al. Tolvaptan, a selective oral vasopressin V2-receptor antagonist, for hyponatremia. N Engl J Med. 2006;355:2099-112. http://dx.doi.org/10.1056/ NEJMoa065181

22. Abreu CC, Fabián MG, Narváez O. Tratamiento actual de la insuficiencia cardiaca aguda descompensada. Revista Mexicana de Cardiología. 2006;17:106-9.

23. Soeder RW. Fern constituents: Including occurrence, chemotaxonomy and physiological activity. Bot Rev. 1985;51:442-536. http://dx.doi.org/10.1007/BF02860970 